\title{
Screening of Bioactive Compounds for Development of New Pesticides: A Mini Review
}

\author{
Shaon Kumar Das \\ ICAR Research Complex for NEH Region, Sikkim Centre, India
}

Copyright $\bigcirc 2016$ by authors, all rights reserved. Authors agree that this article remains permanently open access under the terms of the Creative Commons Attribution License 4.0 International License

\begin{abstract}
With the discovery of combinatorial chemistry, high-throughput screening, chemical genetics, chemoinformatics and gene expression profiling, huge number of synthetic compound libraries have generated and the pace of discovery and screening of pesticides has dramatically escalated significantly and have become modern tools in the burgeoning field of agrochemicals. Today, automated procedures allow more than 5 lakhs of compounds to be screened per year under in-vivo high-throughput screening, which is invaluable in the search for new leads. Natural products from microbes and plants offer a broad array of lead molecules with great diversity in their structures and biological activity. Screening methods for active constituents include bioassay-guided isolation, fractionation-driven bioassay, isolate and assay, and with increasing exploitation of lead based biochemical combinatorial chemistry approaches. The contribution of biorational design is continuously rising to identify novel mode of action in the discovery process. These developments will prompt the agricultural chemists to take a new look at the way chemistry was traditionally done in the laboratory. The objective of this review article is to elaborate different screening procedure for development of new pesticides.
\end{abstract}

Keywords Combinatorial Chemistry, High-throughput Screening, Natural Products, Bioassay, Mode of Action

\section{Introduction}

Extracts of plant products having pesticidal components have been used to different degrees over the years which is often not in a systematic way. Progress in crop protection by chemical has been extraordinary over the last decades, not only in the invention of new and selective active ingredients but also in the assessment of the behavior of these chemicals in the environment. Scientific progress in chemistry, biology, molecular biology, and biotechnology has revolutionized the way of searching for new agrochemicals over the past decade.
Using DDT as an oldest organochlorine insecticide, the plant hormone-based phenoxy-acetic acid derivatives as different herbicides, sometimes carbamates as insecticides, different organophosphates, and and thiram as a fungicide marked- at least in terms of the selective activity in pest controlquantum-leap progress in pest control. We are certainly once again at the beginning of a new technological development for the protection of our traditional crops as well as in the creation of novel foodstuffs. Novel different biotechnological methods to achieve general goals are increasingly becoming available and in future these may become of greater importance, and thus the pure chemical technology of crop protection in agriculture is complementing or replacing ${ }^{[1]}$. But chemical crop protection will try to continue to play a major role in agriculture although of the emergence of new novel biotechnological solutions.

\section{Steps Involve Commercialization of a New Pesticide}

Traditional method of pesticide discovery is based on the synthesis and mass screening of large numbers of compounds. Curiosity in discovering the new chemicals extended more than hundred years. This process relies exclusively on introducing as many as 20,000 compounds may have to be synthesized and examined at a cost of millions of dollars before a pesticide worthy of chemical development is identified which is both expensive and time-consuming. Further directed synthesis then strives to increase the activity of promising leads. All the pesticides in the 1940s like DDT, 2, 4-D, OPs, carbamates, thiram were introduced by these processes. The traditional approaches for discovery and screening of pesticides are Empirical synthesis/conventional screening, Analogue synthesis/ SAR optimization, Natural product models, Biochemical design and, Biorational synthesis/innovative screening. Steps involves in commercialization of new pesticides are presented in Figure 1. 


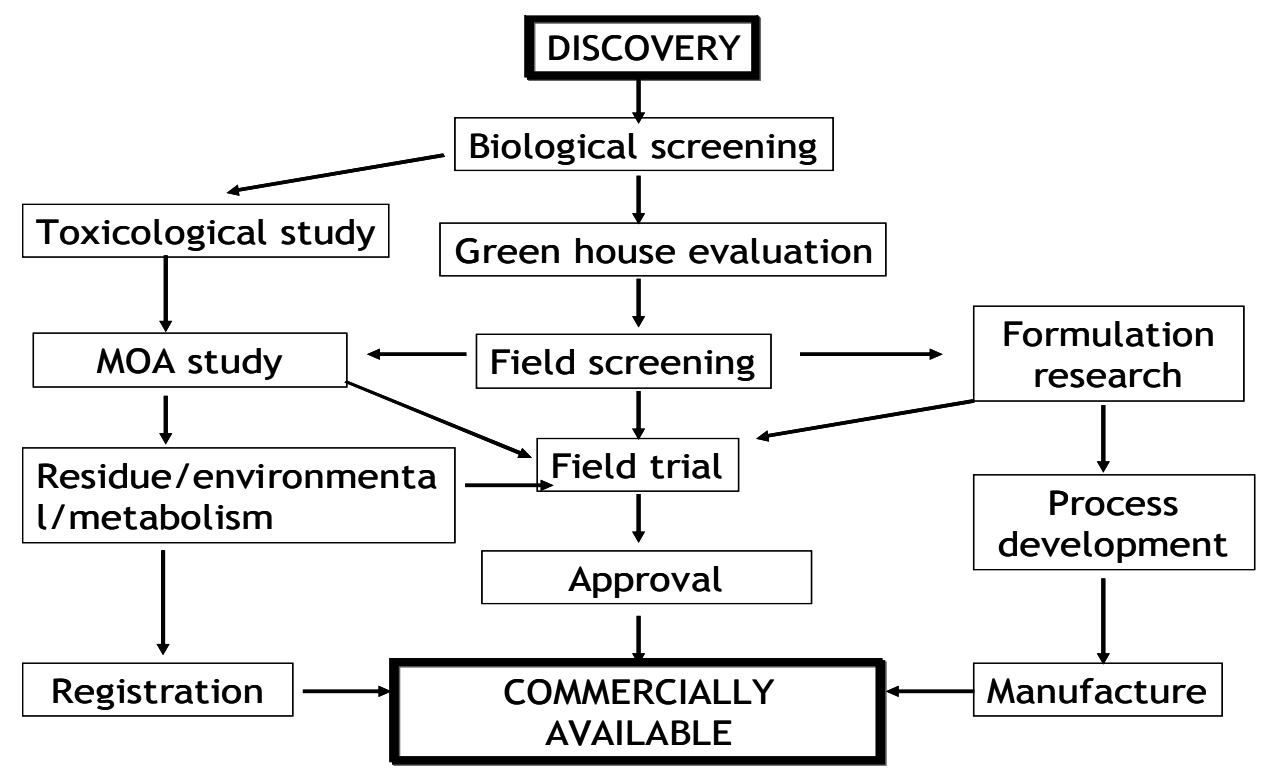

Figure 1. Steps for commercialization of new pesticides

The discovered of pesticide in laboratory to its commercialization process involves many lengthy steps. This is cost effective and time consuming steps again delayed pesticide development process by another three to four years gave a new discovery strategy of pesticide as given below-

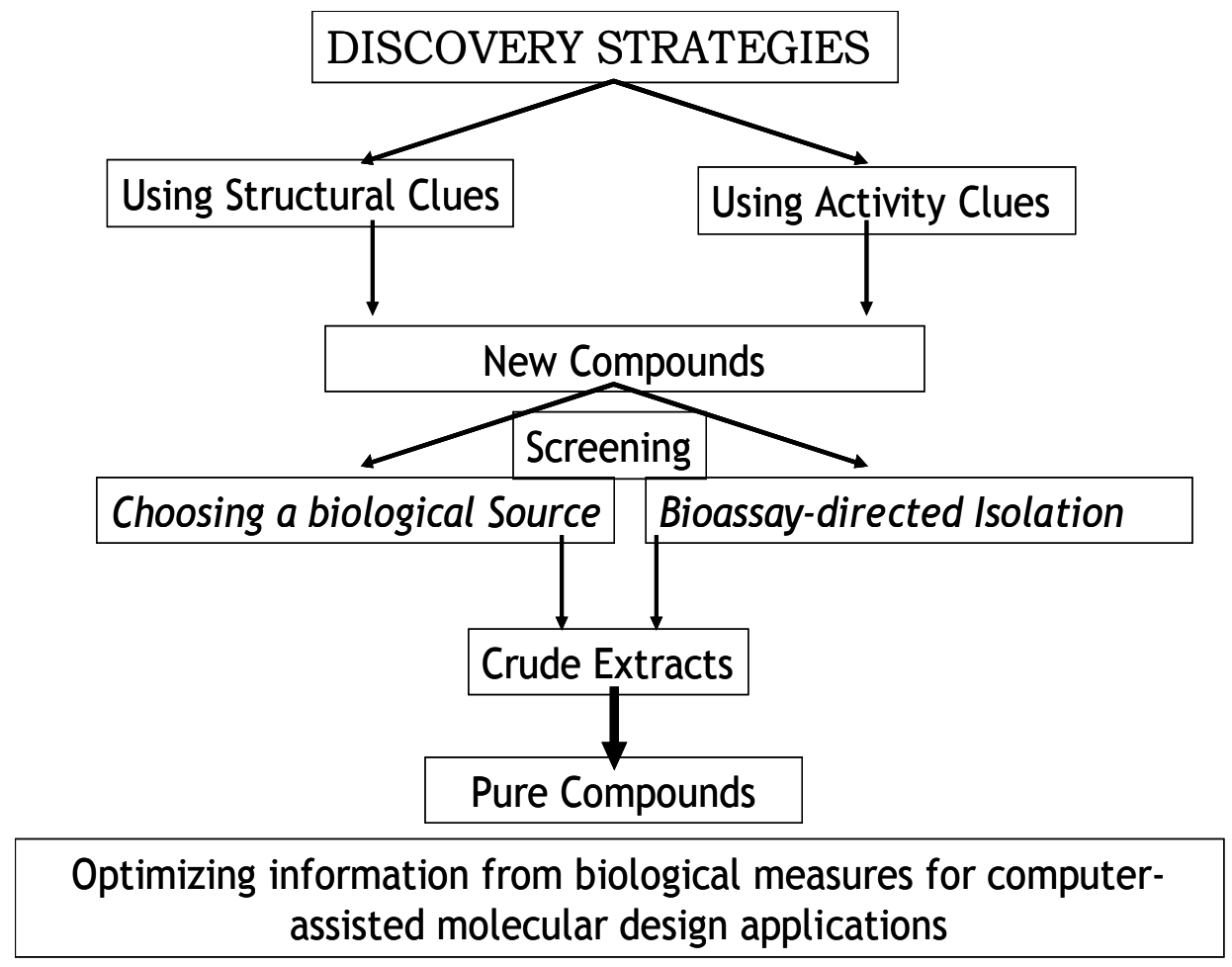

Figure 2. Discovery strategies and computer assisted molecular strategies 


\subsection{Structure Activity Relationship (SAR)}

Further, different methods allowing correlation of biological activity, structural properties and structure activity relationship (SAR), have been identified, developed and refined. Structure-activity-relationship is based on the concept that various substances with similar chemical structure have more or less same biological activity ${ }^{[2]}$. SAR is basically a qualitative comparison of structures of the chemical compounds and various effects in different biological system. SAR can be defined as the study of relationships between molecular structure and physico-chemical and biological responses.

\subsection{Bioassay Screening}

The activity of screening of compounds in natural disease-insect pest/crop-weed complex is by nature relatively easy. This indicate the long practice of growing plants (weed and crop plants) under controlled conditions in the propagating obligate, greenhouse, and non-obligate diseases and rearing insect-pests. The bioassay plays a very important role in natural products chemistry research though it is a conventional method of screening (Table 1). Now a day, new automated procedures allow us more than 100000 compounds to be screened every year under quasi "near field-like" conditions, which is not valuable in the search for new leads compounds.

Table 1. Most commonly used bioassay organisms

\begin{tabular}{|c|c|c|}
\hline Insecticidal assay & Herbicidal assay & Fungicidal assay \\
\hline Diabrotica virgifera & & Stagonospora nodorum \\
Heliothis viresence & Nicotiana tabacum & Fusarium culmorum \\
Lygus hasperus & & Puccinia recondita \\
\hline
\end{tabular}

\subsection{Bioassay Guided Isolation}

Even Bioassay-directed isolation has been one of the preferred methods of discovery after producing organism is selected. This is a relatively new method and could be followed in searching for active constituents from plants. Bioassay-guided isolation of compounds with potential use as pesticides or pesticide leads ${ }^{[3]}$. This type of laborious approach often leads to rediscovery of various known compounds. Modern day's tandem separation or other chemical characterization instrumentation can sufficiently eliminate much of this problem by identification of compounds before their bio-assay.

\subsection{Quantitative Structure-Activity Relationship (QSAR)}

It is the quantitative correlation between the biological (ecological, toxicological or pharmacological) activity to the structure of chemical compound(s), which allows the prediction of the "desire efficacy" of a structurally related compound. As early as 1869, QSARs in environmental toxicology were reported, ${ }^{[4]}$ on narcotics while QSARs in environmental. However, different pesticides are developed based on their specific mode of mechanism of action. Thus, this may affect the predictability of QSARs. An evaluation at this stage may result in a less promising result, as most of the preliminary work has been done on simpler molecules ${ }^{[5]}$.

\subsection{Mode of Action Based Identification of Compounds}

Mode of action (MOA) of a particular class of chemical compounds permits rational design of pesticide through studies of structure-activity relationships and utilized this information about the MOA of the compounds being screened. Although this approach affords a certain advantage by preventing the limitations of our knowledge from constraining the scope of discovery and it is not very efficient. Concept of relative potency was introduced and activity was related to that of the standard in the same test.

\subsection{Discovery of Leads}

Active substances are constantly searching by the chemists for some new sources of attractive lead molecules. These are the substances which already have a particular desired biological effect. But lack certain characteristics for "practical" use as active compounds. The enormous variety in nature offers broad scope for new ideas here.

\subsection{In Vitro High Throughput Screening}

The use of in-vitro assays against molecular targets for the evaluation of chemicals as lead structures in pesticide discovery. New compounds acting on defined or undefined targets can be screened by this method. Development of agrochemical by in vivo screening, more than 5 lakhs of compounds can be screened per year in contrast to less than 10000 compounds per year during 1980s. Modern in Vitro High Throughput Screening instruments are used where more than 100 plates per day for a single point enzymatic assay can be performed with 15 -minute reaction time ${ }^{[6]}$.

\subsection{Combinatorial Chemistry}

The basic idea of combinatorial chemistry is to synthesize rapidly large amounts of different compounds at the same time, using a process that is supported by computation and automation ${ }^{[7]}$. Combinatorial techniques enable chemists to combine several chemical building blocks in many different ways, resulting in large numbers of different compounds. Pharmaceutical and agrochemical companies face considerable pressure to develop new drugs and pesticides in a less costly and time consuming way. Traditionally, chemists in the industry sector had to synthesize possible drug of pesticide candidates one by one before they could screen the activity of the candidate. Combinatorial chemistry provides a tool to meet these demands for cuts in costs and time ${ }^{[8]}$. Large numbers of compounds are now available 
through combinatorial chemistry to screen for lead-level agrochemical activity. Compounds with whole-organism activity can be discovered if compounds with certain ranges of simple physicochemical parameters consistent with transport to the target site (like molecular mass, log $\mathrm{P}$, hydrogen-bond donors and acceptors, rotatable bonds) are selected for screening. The distribution of these parameters for commercial insecticides and post-emergence herbicides was examined and ranges consistent with whole-organism activity are proposed for the two classes of agrochemical ${ }^{[9]}$. It offers rapid, productive and cost-efficient generation of compounds and routine method for the discovery and optimization of lead molecule. Applications in drug development, small molecule libraries, molecular diversity allow solution and solid-phase synthesis.

\subsection{High Throughput Screening (HTS)}

High throughput is an adjective use before screening to become fastest - first and best. It represents high speed of screening throughout process and reflects what chemists can easily work upon. Use of in-vitro and in vivo assays against molecular targets for the evaluation of chemicals as lead structures in pesticide discovery ${ }^{[10]}$. Traditional in vivo screening processes are time consuming, laborious, slow in process, requires larger space. In vivo high throughput screening permits rapidly screen large amounts of compounds for biological activity. The natural substance extracts are then automatically tested by ultra-high-throughput in vivo screening (UHTVS), which is performed for appropriate insecticidal, fungicidal and herbicidal efficacy. If the robots discover that a sample has the desired biological effect, it goes through further stages of isolation before being tested for its suitability as a possible pesticide.

\subsection{Virtual Target Based Screening}

Virtual target based screening allows for millions of chemical compounds to be computationally selected based on structural complimentary to known inhibitors or to a target binding site on a biological macromolecule. Compound selection in virtual screening (VS) targeting a biological macromolecule is typically based on the interaction energy between the chemical compound and the target macromolecule ${ }^{[11]}$. It is used as guidance for chemical virtual synthesis and virtual screening simultaneously in computer-aided molecule design. The in silico procedure of virtual screening (VS) and its relationship to the experimental procedure, HTS, new developments in the field of drug and pesticide development and perspectives on future research are offered. There are several methods for virtual screening of organic compounds concentrated on screening by docking and by pharmacophore searching. To study the utility of the virtual combinatorial chemistry coupled with computational screening, a library of amine and urea derivatives was designed by virtual combinatorial synthesis and eventually computationally screened by a mathematical topological model as antihistaminic compounds. The results reveal that virtual combinatorial synthesis and virtual screening together with molecular topology is a powerful tool in the design of new drugs ${ }^{[12]}$.

\subsection{Some Application of HTS in Agrochemical Discovery}

New classes of fungicides viz. strobilurins, phenylpyrroles, anilinopyrimidines, phenoxyquinolines, have been identified through the development of HTS comprised of target enzyme sites or cell-based assays ${ }^{[13]}$. Library of 1126 diverse amides substituted bicycle [2, 2, 2]-octenones was screened in HTS in vivo against a number of weed, insect and fungal model organisms leading to the discovery of a novel series of herbicidally active compounds ${ }^{[14]}$.

\subsection{Chemical Genetics}

The term chemical genetics was first used in the scientific literature in 1935 by von Euler et al. published work on the "chemical genetics of chlorophyll mutating barley strains" obtained through spectroscopic analyses of metabolites of selected barley (Hordeum vulgare) strains. From then until the late 1980s the term chemical genetics was used mainly to describe studies of the differences in chemical constitution between mutant strains of various organisms, the majority of which were plants ${ }^{[15]}$. Chemical genetics reemerged on the scientific landscape with force in the middle to late 1990s, when recognized the potential power of the systematic use of small molecules to address questions in cell biology. It provides efficient identification of both candidate compounds and their targets. They termed this general approach to biology chemical genetics, of which the goal is the delineation and characterization of signaling pathways for particular gene products using cell-permeable small molecules as modulating ligands.

\subsection{Competitive Indirect-ELISA (CI ELISA)}

It is an antibody based approach for screening. Antibodies are mimics of biological receptors and it can be used as surrogate of pesticide target sites. An immunological ciELISA was developed to determine the potential inhibitor of acetyl coenzyme-A carboxylase (ACCase) specific monoclonal antibodies as screening tools to identify novel lead chemistry. Cyclohexanediones,

aryloxyphenoxypropionates, indolizidinediones, and triazinediones are four known structural classes of herbicides that inhibit ACCase ${ }^{[17]}$. Two monoclonal antibodies $\mathrm{mAb} A$ and $\mathrm{mAb} \mathrm{B}$ and three different cyclohexanedione hapten coating conjugates were reported to very promising ${ }^{[16]}$. In another study, on the basis of $\mathrm{IC}_{50}$ value, they separate 24 cyclohexanediones analogues into active and inactive 
ACCase inhibitors, suggesting that both indolizidinediones and cyclohexanediones possess features recognized by monoclonal antibodies important for the inhibition of ACCase activity. In conclusion, for the identification of new lead chemicals in a pesticide discovery program, different pharmacophore-specific antibodies may be potentially valuable screening tools.

\subsection{Chemoinformatics}

It is the science of application of information technology to problems in chemistry through management, analysis, and dissemination of data related to chemical compounds. The use of different information technology and management practices has become a critical part of the toxicity prioritization \& screening process. Thus, chemoinformatics is mixing of those information resources to transform different data into information and finally, information into knowledge. The objective is for making better decisions faster in the area of activity screening (Weber et al., 2001). In fact, Chemoinformatics is a generic term which encompasses the organization, management, retrieval, design, creation, visualization, analysis, dissemination, and use of different chemical information. It transforms data into information and subsequently into knowledge, thus greatly facilitating all aspects of chemical research. In summary, chemoinformatics has become one of the essential tools in the early stages of pharmacological as well as in agrochemical discovery during the last decade ${ }^{[18,19],[20]}$. The main reason for its importance in modern days is rooted in the emergence of high throughput chemical synthesis and high throughput screening as a dominant technology for the discovery of starting points for chemical optimization.

\subsection{Other Modern Techniques for Discovery and Screening of Pesticide}

Chemists employ molecular modeling, using the information from different spatial structure of a molecule, to analyze a substance and to create related substances by different computer simulation. Microderivatizing- a technique is use in chemical processing to alter the structural parts of the natural substance molecule and test whether these are still biologically active and devise simpler molecular structures which ideally have the same or even an improved effect can be synthesized.

\section{Conclusions}

With the advent of combinatorial chemistry and HTPS techniques over the past decade significantly larger synthetic compound libraries have been generated. Today, automated procedures allow more than 5 lakhs compounds to be screened per year under in vivo HTPS, which is invaluable in the search for new leads. The pace of discovery and screening of pesticides has dramatically escalated by the use of chemical genetics and chemo informatics. Development of recombinant DNA technology, identify genes that are expressed during infestation of obligate biotrophs. The contribution of biorational design is continuously rising to identify novel MOA in the discovery process. New agrochemicals have discovered by the process of screening of natural products, with increasing exploitation of lead based and bioassay guided isolation.

\section{REFERENCES}

[1] Bratman S. and Girman. (2003). A Hand book of Herbs and Supplement and their Therapeutic Uses. St. Louis: Mosby, 769-771.

[2] Das S. K., \& Mukherjee I. (2011). Effect of light and pH on persistence of flubendiamide. Bull Environ Contam Toxicol, $87,292-296$.

[3] Das S. K., \& Mukherjee I. (2012). Effect of moisture and organic manure on persistence of flubendiamide in soil. Bull Environ Contam Toxicol, 88, 515-520.

[4] Das S. K., Mukherjee I., \& Das S. K. (2012). Dissipation of flubendiamide in/on Okra [Abelmoschus esculenta (L.) Moench] Fruits. Bull Environ Contam Toxicol, 88, 381-384.

[5] Das S. K., \& Mukherjee I. (2012). Flubendiamide Transport Through Packed Soil Columns. Bull Environ Contam Toxicol, 88, 229-233.

[6] Das S. K., \& Mukherjee I. (2014). Influence of microbial community on degradation of flubendiamide in two Indian soils. Environ Monitor \& Assess, 186, 3213-3219.

[7] Das S. K., Mukherjee I., and Kumar A. (2015). Effect of soil type and organic manure on adsorption-desorption of flubendiamide. Environ Monitor \& Assess, 187:403. DOI 10.1007/s 10661-015-4623-2.

[8] Das S. K., and Avasthe R. K. (2015). Biochar as carbon negative in carbon credit under changing climate. Current Science, 109, 1223.

[9] Duart M. J., R. Garcia-Domenech, G. M.Anton-Fos, and J. Galvez (2001). Optimization of a mathematical pattern for the prediction of antihistaminic activity. J. Comput. Aid. Mol. Design, 15, 561-572.

[10] Duke J., and M. Bogenschutz (2002). Duke Hand book of medicinal Plants. $2^{\text {nd }}$ Boca Raton, FL: CRC Press; 529.

[11] Galvez J., R. Garcia-Domenech, and J. V. de Julian-Ortiz (2005). Some relationships between molecular energy-topology and symmetry. Fundamentals of molecular similarity, Kuwer Academic/Plenum Publishers (Ed. R. Carbo-Dorca), 11-31.

[12] Garcia-Domenech R., J. V. de Julian-Ortiz, and M. J. Duart (2001). Search of a topological pattern to evaluate toxicity of heterogeneous compounds. SAR and QSAR in Envir. Res., $12,237-254$.

[13] Hansch C., and A. Leo (1995). Exploring QSAR. Fundamentals and Applications in Chemist $\mathrm{O}^{\prime}$ and 3 ioloRv, American Chemical Society. 
[14] Lahuerta L., Y. Fuster, M. Duart, G. M. Anton, R. Garcia-Domenech, J. Galvez, and M. Martinez (2001). Prediction of the chemiluminiscent behavior of pharmaceuticals and pesticides. Anal. Chem., 73, 4301-4306.

[15] Lindell S. D., and J. Scherkenbeck (2005). Prospects for combinatorial chemistry in the agro-sciences. Comb Chem High Throughput Screen. 8, 555-62.

[16] Mishra R., R. Garcia-Domenech, and J. Galvez (2001). Getting discriminant functions of antibacterial activity from physic-chemical and topological parameters. J. Chem. Inf. Comput. Sci., 41, 387-393.

[17] Rimando, A. M., M. Olofsdotter, and S. O. Duke, (2001). Searching for rice allelochemicals. Agron. J., 93, 16-20.
[18] Scherkenbeck J., and S. Lindell (2005). Applications of combinatorial chemistry in the agro-sciences. Comb Chem High Throughput Screen. 8, 563-76.

[19] Sells M. A., J. T. Boyd, and J. Chernoff (1999). P 21-activated kinase $1(\mathrm{Pak} 1)$ regulates cell motility in mammalian fibroblasts. J. Cell Biol. 145, 837-49.

[20] Weber R. J., G. Chen, D. D. Davis, R. L. Mauldin, D. J. Tanner, F. L. Eisele, A. D Clarke, D. C. Thornton, and A. R. Bandy (2001). Measurements of enhanced $\mathrm{H}_{2} \mathrm{SO}_{4}$ and $3-4 \mathrm{~nm}$ particles near a frontal cloud during the First Aerosol Characterization Experiment (ACE 1), J. Geophys. Res., 106(D20), 24, 107-117. 